\title{
SERIES SOLUTION OF FRACTIONAL PANTOGRAPH EQUATIONS VIA TAYLOR SERIES
}

\author{
Amber Shaikh \\ National University of Computer and Emerging Sciences. Karachi (Pakistan) \\ E-mail: amber.shaikh@nu.edu.pk \\ Fozia Hanif \\ University of Karachi. Karachi (Pakistan) \\ E-mail:ms_khans2011@hotmail.com \\ M. Sadiq Ali Khan \\ University of Karachi. Karachi (Pakistan) \\ E-mail:msakhan@uok.edu.pk
}

Asif Jamal

Federal Urdu University of Art And Science Technology \& Department of Basic Science DHA Suffa University. Karachi (Pakistan)

E-mail: asifjamal46@yahoo.com

\begin{abstract}
Hassan Khan
Bahria University. Islamabad (Pakistan)

E-mail: hassan_khan94@hotmail.com
\end{abstract}

Saqib ur Rehamn

Bahria University. Karachi (Pakistan)

E-mail: saqib.rehman@uok.edu.pk

Recepción: 05/03/2019 Aceptación: 12/04/2019 Publicación: 17/05/2019

\section{Citación sugerida:}

Shaikh, A., Hanif, F. Ali Khan, M. S., Jamal, A., Khan, H. y ur Rehamn, S. (2019). Series solution of fractional Pantograph equations via Taylor series. 3C Tecnología. Glosas de innovación aplicadas a la pyme. Edición Especial, Mayo 2019, pp. 322-349. doi: http:// dx.doi.org/10.17993/3ctecno.2019.specialissue2.322-349

\section{Suggested citation:}

Shaikh, A., Hanif, F. Ali Khan, M. S., Jamal, A., Khan, H. \& ur Rehamn, S. (2019). Series solution of fractional Pantograph equations via Taylor series. $3 C$ Tecnología. Glosas de innovación aplicadas a la pyme. Special Issue, May 2019, pp. 322-349. doi: http://dx.doi. org/10.17993/3ctecno.2019.specialissue2.322-349 


\section{ABSTRACT}

This article is devoted to develop a numerical approximation called Taylor minimization method for initial and boundary value fractional Pantograph equations, which governs the modelling of the train system, with neutral and multi-term delays. Taylor optimization technique is basically composed of truncated Taylor series approximation of unknown function while employment of procedure is accompanied by an optimization strategy that is simulated annealing for carrying out the learning phase of unknown Taylor series coefficients. The proposed technique is implemented on various models of Pantograph equations to study the applicability and effectiveness of the planned scheme while error analysis and comparison with previous methods are performed to validate the results. To measure the capability of convergence the data for 100 numbers of independent runs is demonstrated in the form of pictorial presentation.

\section{KEYWORDS}

Taylor series, Fractional Pantograph equation, Simulated annealing, Proportional delays. 


\section{INTRODUCTION}

Several physical phenomena in diverse research areas are modeled in the form of fractional order differential or integro differential equations. For instance, to describe the viscid interactions in human cancellous bone by using Biot's theory (Sebaa, et al., 2006), study of cardiac tissue by using electrode interface (Magin \& Ovadia, 2008), for comprehensive study of vestibule-ocular reflex model (Robinson, 1981), modeling of phenomena in fluid mechanics (Kulish \& Lage, 2002) and introducing fractional order impedance in electric circuits (LeMahute \& Crepy, 1983; Kaplan, Gray \& Liu, 1987). Due to the diverse applications of fractional calculus in real life phenomena, numerous numerical techniques have been developed to solve the fractional order differential and integral equations (Arikoglu \& Ozkol, 2007; Diethelm, Ford \& Freed, 2002; Bai \& Lü, 2005; Agarwal, Lakshmikantham \& Nieto, 2010).

In this effort, we are developing Taylor minimization method (TMM) based on Taylor series. Taylor series is a useful tool for attaining the approximate solution of differential equations on a continuous domain. Various methods have been developed to investigate the solution of differential equations (DEs) by Taylor series. A computational scheme for converting Partial differential equations (PDEs) into algebraic equations based on Taylor series expansion was developed in (Groza \& Razzaghi, 2013). Singular boundary value problems with exponential nonlinearity were solved effectively by using the Taylor series method (Chang, 2014). Some other notable methods of obtaining the solution of delay systems by employing Taylor series can be seen in (Marzban \& Razzaghi, 2006; Razzaghi \& Razzaghi, 1989). But here we are using optimization strategy i.e. simulated annealing (SA) to solve the minimization problem formed by Taylor series approximation of fractional differential equations (FDEs). Simulated annealing is a meta heuristic algorithm inspired by the cooling schedule of metals. Advantage of using this method is its strong ability of escaping from local minima and acquiring global minima with efficiency (Kirkpatrick, Gelatt \& Vecchi, 1983). To demonstrate the applicability and efficiency of method simulation is carried out for fractional pantograph equation in (Rahimkhani, Ordokhani \& Babolian, 2018). 


$$
D^{\alpha} f(t)=f(t)+\sum_{n=1}^{l} f_{n}(t) D^{\alpha n} f\left(q_{n} t\right), \quad m-1<\alpha \leq m, \mathrm{~m} \in \mathrm{N}, t \in[0, h]
$$

subject to the initial conditions.

$$
f^{(i)}(0)=\beta_{i}, \quad i=0,1, \ldots, m-1
$$

It is a functional differential equation with proportional delays. Due to its vast applications in science and engineering it took the attention of many researchers to propose numerous schemes for the simulation of Pantograph equation (Rahimkhani, et al., 2018; Iqbal, Saeed \& Mohyud-Din, 2015; Raja, 2014; Bharawy, Al-Zahrani, Alhamed \& Baleanu, 2014; Saadatmandi \& Dehghan, 2009; Isah, Phang \& Phang, 2017; Syam \& Jaradat, 2017).

In the proposed scheme, the unknown function is approximated in terms of Taylor series which will be transformed into a trial solution by imposing initial and boundary conditions. The same procedure will be adopted for delay terms. Later required derivatives of trial solution, delay trial solution and a trial solution will be substituted in the differential equation to calculate MSE or fitness function with the help of trial points. The substitution will give rise to a minimization problem that was solved by simulated annealing for unknown Taylor series coefficients. Later the coefficients will be utilized to find the Taylor series of unknown function at the domain $[0, h]$. To demonstrate the efficiency of employed scheme various numerical experiments of fractional pantograph equations have been discussed for error analysis and numerical simulation.

\section{PRELIMINARIES}

In the following section we are introducing some basic definitions regarding fractional calculus and Taylor series. 


\subsection{DEFINITION No1}

If a function $\varphi$ is defined on interval $[\alpha, \beta]$ and holds the following conditions

- $\left(\varphi^{(k-1)}\right)$ is continuous on $[\alpha, \beta]$

- $\varphi^{k}$ exist on $] \alpha, \beta[$

and $x \in[\alpha, \beta]$ then from (Malik \& Arora, 1992)

$$
\varphi(x)=\left(\sum_{\mu=0}^{k-1} \frac{\varphi^{(\mu)}(\alpha)(x-\alpha)^{(\mu)}}{\mu !}\right)+\frac{(x-\alpha)^{(k)}(1-\theta)^{k-p}}{p[(k-1) !]} \varphi^{(k)}(c)
$$

where $0<\theta<1, p>0$ and $\alpha<c<x$. For $\alpha=0$ and truncated to $k$ th term Eq. (2) can be expressed as

$$
\varphi(x)=\left(\sum_{\mu=0}^{k-1} \frac{\varphi^{(\mu)}(0)(x)^{(\mu)}}{\mu !}\right)
$$

with Lagrange's form of the remainder after $k$ terms as

$$
R_{n}=\frac{(x)^{k}}{k !} \varphi^{(k)}(\theta \mathrm{x})
$$

\subsection{DEFINITION No 2}

Fractional derivative in sense of Caputo for the order $\kappa \geq 0$ can be described as in (Heydari, et al., 2015)

$$
D^{\kappa} f(t)=\frac{1}{\Gamma(m-\kappa)} \int_{0}^{t}(t-s)^{m-\kappa-1} f^{(n)}(s) d s, m-1<\kappa<m, \mathrm{~m} \in \mathrm{N} .
$$

with the fulfillment of following properties

$$
\begin{aligned}
& D^{\kappa} I^{\kappa} f(t)=f(t), \\
& I^{\kappa} D^{\kappa} f(t)=f(t)-\sum_{j=0}^{m-1} f^{(j)}(0) \frac{x^{j}}{j !},
\end{aligned}
$$

- $D^{\kappa} c=0$, where $\mathrm{c}$ is a constant 
- $D^{\kappa}\left(t^{\beta}\right)= \begin{cases}0, & \kappa \in \mathrm{N}_{0}, \beta<\kappa \\ \frac{\Gamma(\beta+1)}{\Gamma(\beta+1-\kappa)} t^{\beta-\kappa}, & \text { otherwise }\end{cases}$

\section{TAYLOR MINIMIZATION METHOD}

\subsection{TAYLOR SERIES APPROXIMATION}

The Taylor series expansion of an analytic function $f(t)$ about $t=0$ upto $\eta$ terms can be given as:

$$
\begin{gathered}
f(t)=\left.\sum_{k=0}^{\eta} \frac{d^{k} f(t)}{d t^{k}}\right|_{t=0} \frac{t^{k}}{k !} \\
f(t)=f(0)+\mathrm{t} f^{\prime}(0)+\frac{t^{2}}{2 !} f^{\prime \prime}(0)+\left.\sum_{k=3}^{\eta} \frac{d^{k} f(t)}{d t^{k}}\right|_{t=0} \frac{t^{k}}{k !}
\end{gathered}
$$

while Taylor series expansion of an analytical function with delay can be expressed as:

$$
\begin{gathered}
f(q t)=\left.\sum_{k=0}^{\eta} \frac{d^{k} f(q t)}{d t^{k}}\right|_{t=0} \frac{t^{k}}{k !} \\
f(q t)=f(0)+q \mathrm{t} f^{\prime}(0)+\frac{q^{2} t^{2}}{2 !} f^{\prime \prime}(0)+\left.\sum_{k=3}^{\eta} \frac{d^{k} f(t)}{d t^{k}}\right|_{t=0} \frac{q^{k} t^{k}}{k !}
\end{gathered}
$$

the generalized fractional pantograph equation can be written as:

$$
D^{\alpha} f(t)=f(t)+\sum_{n=1}^{l} f_{n}(t) D^{\alpha n} f\left(q_{n} t\right), \quad m-1<\alpha \leq m, \mathrm{~m} \in \mathrm{N}, t \in[0, h]
$$

Subject to the initial conditions and boundary conditions:

$$
\begin{aligned}
& f^{(i)}(0)=\beta_{i}, \quad i=0,1, \ldots, m-1 \\
& f^{(i)}(\alpha)=\alpha_{i}, \quad i=0,1, \ldots, m-1
\end{aligned}
$$


Now generate the trial solution by substituting values from Eq. (7) and Eq. (8) in Eq. (3) and Eq. (5). For $i=0$ in Eq. (3) and $i=1$ in Eq.(5):

$$
f(t)=\beta_{0}+\mathrm{t} f^{\prime}(0)+\frac{t^{2}}{2 !} f^{\prime \prime}(0)+\left.\sum_{k=3}^{\eta} \frac{d^{k} f(t)}{d t^{k}}\right|_{t=0} \frac{t^{k}}{k !}
$$

for introducing boundary conditions put $t=\alpha$ in Eq.(9):

$$
f(\alpha)=\beta_{0}+\alpha f^{\prime}(0)+\frac{\alpha^{2}}{2 !} f^{\prime \prime}(0)+\left.\sum_{k=3}^{\eta} \frac{d^{k} f(t)}{d t^{k}}\right|_{t=0} \frac{\alpha^{k}}{k !}
$$

substituting values from Eq.(8) we get:

$$
\alpha_{1}=\beta_{0}+\alpha f^{\prime}(0)+\frac{\alpha^{2}}{2 !} f^{\prime \prime}(0)+\left.\sum_{k=3}^{\eta} \frac{d^{k} f(t)}{d t^{k}}\right|_{t=0} \frac{\alpha^{k}}{k !}
$$

solve Eq. (11) $f^{\prime}(0)$ and substitute it in Eq. (9) we get:

$$
f(t)=\beta_{0}+\frac{\mathrm{t}}{\alpha}\left(\alpha_{1}-\beta_{0}-\frac{\alpha^{2}}{2 !} f^{\prime \prime}(0)-\left.\sum_{k=3}^{\eta} \frac{d^{k} f(t)}{d t^{k}}\right|_{t=0} \frac{\alpha^{k}}{k !}\right)+\frac{t^{2}}{2 !} f^{\prime \prime}(0)+\left.\sum_{k=3}^{\eta} \frac{d^{k} f(t)}{d t^{k}}\right|_{t=0} \frac{t^{k}}{k !}
$$

Eq. (9) will be trial solution for initial value problem while Eq. (12) will be trial solution for boundary value problem. For obtaining delay trial solution same procedure will be adopted for Eq. (5). Substitute the values of trial solution, delay trial solution and their required derivatives by definition No 2 in Eq.(6) and MSE as:

$$
M S E=\sum_{j=0}^{\gamma}\left(D^{\alpha} f\left(t_{j}\right)-f\left(t_{j}\right)-\sum_{n=1}^{l} f_{n}\left(t_{j}\right) D^{\alpha n} f\left(q_{n} t_{j}\right)\right)^{2}, m-1<\alpha \leq m, \mathrm{~m} \in \mathrm{N}, t \in[0, h]
$$

After discretizing the domain the differential equation has been transformed into a minimization problem that can be solved by simulated annealing to obtain the values of unknown $f^{(i)}(0)$ that can be substituted in Eq. (1) to get the required solution. The procedure can be further analyzed through Figure 1.

\subsection{SIMULATED ANNEALING}

Process of Simulated annealing is a generic probabilistic meta-algorithm that is an advancement of Genetic algorithms to improve the convergence of procedure and to avoid local optima with simple applicability. Here the objective function 
is the MSE of fractional Pantograph delay differential equation. Following parameters are marked noteworthy in the implication of procedure.

$T_{K}$ represents the value of temperature at the end of each iteration

- while $T_{0}=1$. At the end of each iteration value of $T$ will be changed by the following way $T_{K}=\chi T_{K-1}, \chi \in[0.8,0.99], k>0$

- $P_{a}=\left\{\begin{array}{cc}e^{\frac{-\rho \nabla \delta}{T}}, & \nabla \delta>1 \\ 1, & \nabla \delta<1\end{array}\right.$, represents the probability of acceptance.

$\nabla \delta$ is the difference between solution errors of consecutive perturbations.

- $\rho=\frac{1}{k_{b}}$, where $k_{b}$ is the Boltzman constant. Boltzman constant is commonly replaced by 1 from the probability of acceptance when there is no need to cope with different materials.

Simulated annealing algorithm is initiated by a random solution guess and a high temperature usually 1 . The energy of the system is calculated at random guess and then again at random neighboring solution. The neighbouring solution is accepted using the probability returned by the above formula by comparing it with a random number ranged between 0 and 1 . The temperature is declined each time, as described above, after processing a certain number of iterations at each temperature value. This is repeated until the system freezes into a steady state. With a declining temperature value of the system, the probability of accepting a worse move is also reduced. Procedure is performed here by Mathematica 11 with default options provided by the software. 


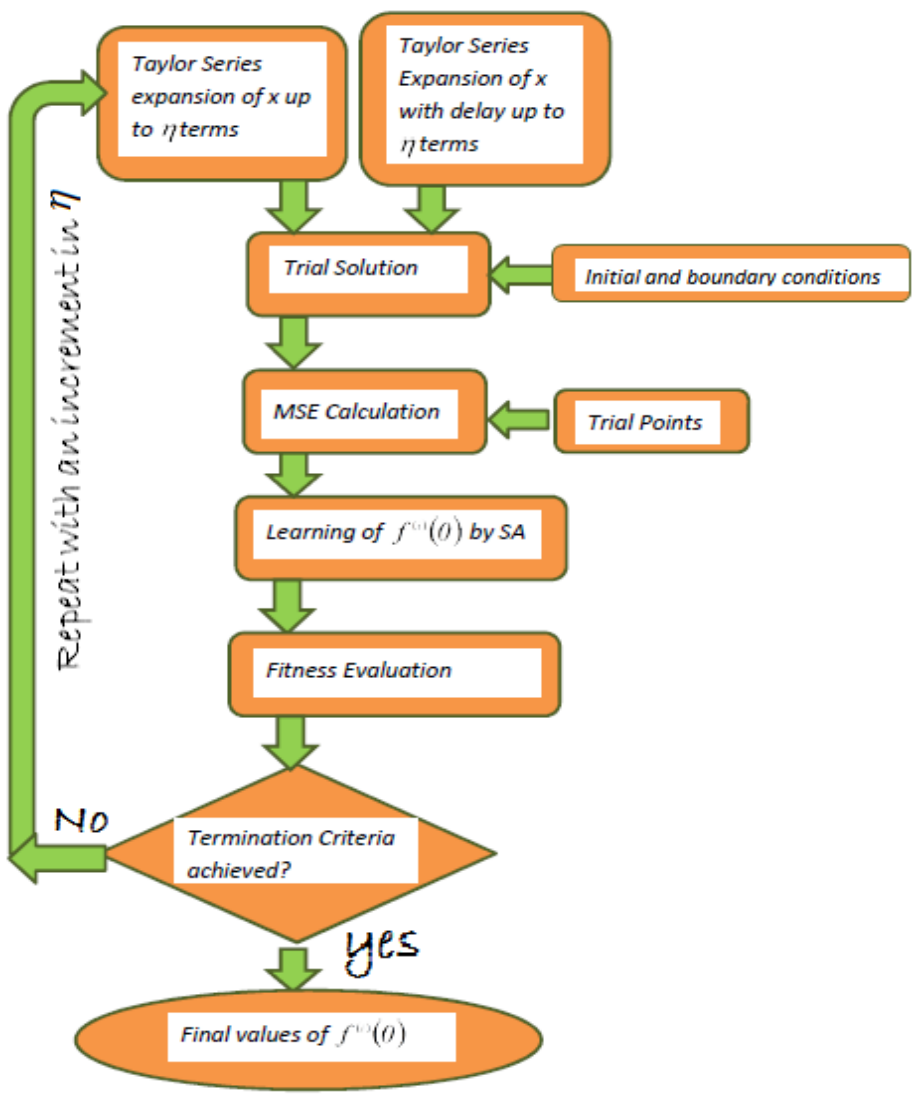

Figure 1. Flow chart of the algorithm.

\section{RESIDUAL ERROR}

For FDEs the exact solution is generally not known so to demonstrate the efficiency of the proposed technique here we are analyzing the error of numerical experiments by the following procedure. Since the approximated solution of fractional pantograph equation can be given by Eq. (3) substituting the obtained values of $\mathrm{f}^{(\mathrm{i})}(0), f(t), D^{\alpha} f(t)$ and $D^{\alpha k} f(t)$ for $\mathrm{k}=1,2,3, \ldots, 1$ in generalized fractional pantograph equation the obtained result at $t \in[0, h]$ will be approximately equal to zero that can be represented by: 


$$
\operatorname{Er}(t)=\left|D^{\alpha} f(t)-f(t)-\sum_{n=1}^{l} f_{n}(t) D^{\alpha n} f\left(q_{n} t\right)\right| \cong 0
$$

While $f(t)$ is the obtained approximated continuous solution by TMM. $\operatorname{Er}\left(t_{i}\right) \rightarrow 0$ as the value of MSE obtained by TMM is sufficiently small enough. The error analysis for each numerical experiment will be performed by the above equation. The convergence of TMM is completely dependent on the convergence of simulated annealing. If $M S E \rightarrow 0$ as $j \rightarrow \gamma$ the Eq. (2) will converge.

\section{NUMERICAL EXPERIMENTS:}

\section{Experiment No 1}

Consider the following linear fractional Pantograph equation with delay,

$$
D^{\lambda} f(t)=\frac{3}{4} f(t)+f\left(\frac{1}{2} t\right)-t^{2}+2, \quad 1<\lambda \leq 2, \quad \mathrm{f}(0)=0, \quad \mathrm{f}^{\prime}(0)=0
$$

By applying the TMM on the above linear pantograph fractional differential model, for $\lambda=2$ and $\mathrm{k}=3$, unknown coefficients are found to be 2 and $3.95 \times 10^{-17}$. By substituting these values in trial solution the required solution is found to be $f(t)=t^{2}+0.67 \times 10^{-16} t^{3}$ which is approximately equal to the true solution $f(t)=t^{2}$. Figure 2 is exhibiting solution of experiment No 1 for $\lambda=1.7,1.8,1.9,2$ and exact solution for $\lambda=2$. Final values of Taylor coefficients can be visualized in pictorial form for different fractional order derivatives in Figure 3 while residual error according to section 4 and MSE for different fractional values of derivatives on the domain of $[0,10]$ can be grasped through Table 1. For ascertaining the efficacy and power of offered scheme, data of 100 numbers of independent runs of the algorithm by altering the perturbation scale can be envisioned through Figure 4. 


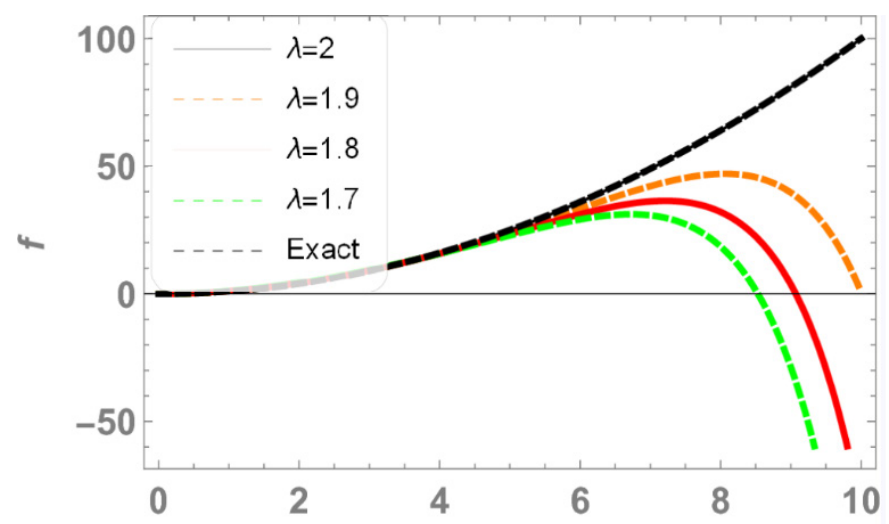

Figure 2. Solution of Example 1 at different values of $\lambda^{t}$.

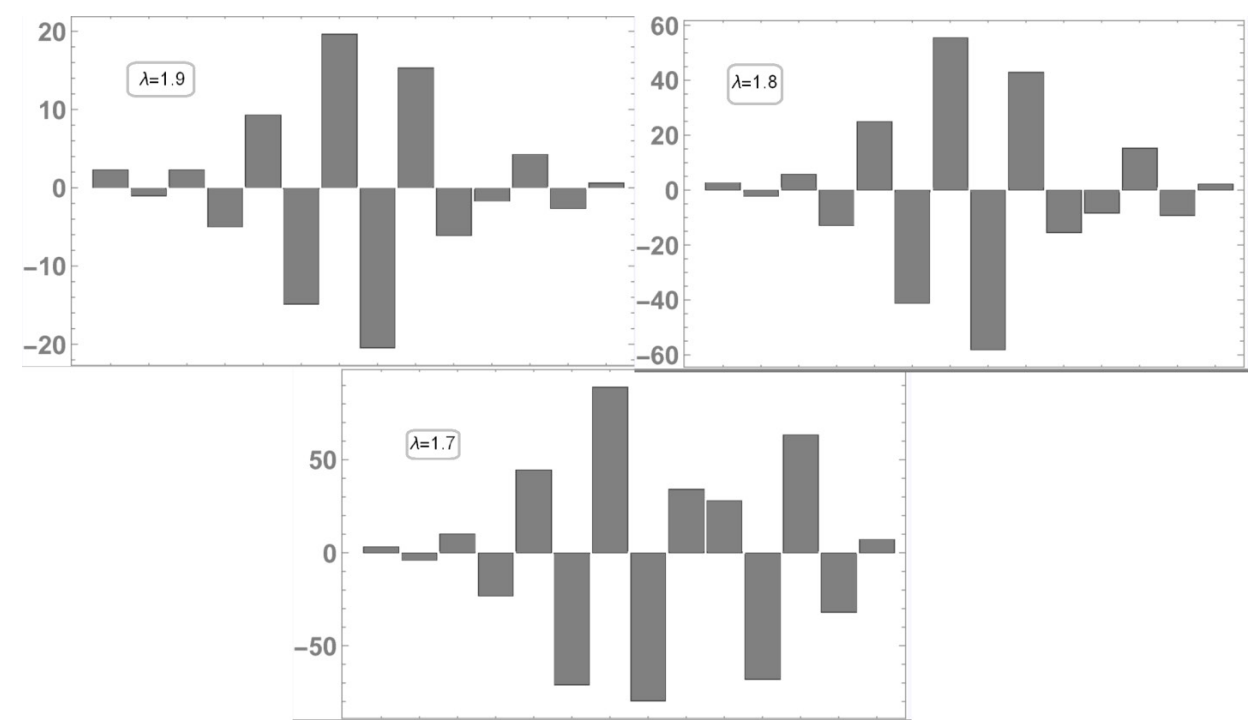

Figure 3. Final values of Unknown Taylor coefficients, for example, No 1 for $k=15$. 

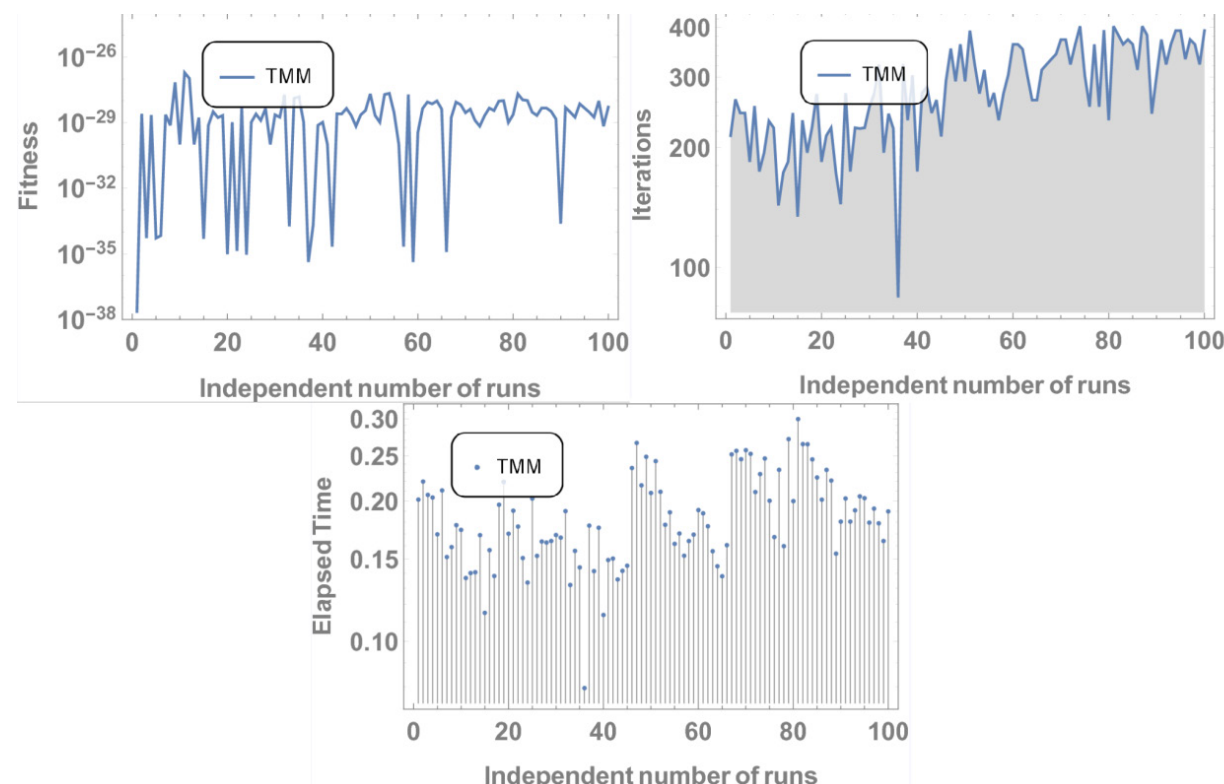

Figure 4. Data of 100 number of Independent runs of example No 1 for $\lambda=2$.

Table 1. Residual error for example No 1.

\begin{tabular}{|c|c|c|c|}
\hline$t$ & $\begin{array}{c}\text { Residual } \\
\text { error }\end{array}$ & $\begin{array}{c}\text { Residual } \\
\text { error }\end{array}$ & $\begin{array}{c}\text { Residual } \\
\text { error }\end{array}$ \\
\hline 1 & $\lambda=1.9$ & $\lambda=1.8$ & $\lambda=1.7$ \\
\hline 2 & $1.4885 \times 10-5$ & $3.6715 \times 10-5$ & $1.1074 \times 10-4$ \\
\hline 3 & $7.4028 \times 10-5$ & $1.9065 \times 10-4$ & $4.3967 \times 10-4$ \\
\hline 4 & $2.9014 \times 10-5$ & $7.4914 \times 10-5$ & $2.0889 \times 10-4$ \\
\hline 5 & $2.0656 \times 10-5$ & $5.3673 \times 10-5$ & $1.5509 \times 10-4$ \\
\hline 6 & $2.1182 \times 10-5$ & $5.9538 \times 10-5$ & $1.5409 \times 10-4$ \\
\hline 7 & $2.4591 \times 10-5$ & $6.8656 \times 10-5$ & $1.5993 \times 10-4$ \\
\hline 8 & $2.5200 \times 10-5$ & $7.2173 \times 10-5$ & $1.5883 \times 10-4$ \\
\hline 9 & $2.0192 \times 10-5$ & $5.8111 \times 10-5$ & $1.3101 \times 10-4$ \\
\hline 10 & $2.2069 \times 10-5$ & $6.8332 \times 10-5$ & $1.2009 \times 10-4$ \\
\hline MSE & $7.3279 \times 10-7$ & $2.3416 \times 10-5$ & $3.7734 \times 10-6$ \\
\hline & $1.9065 \times 10-10$ & $1.4138 \times 10-9$ & $6.6420 \times 10-9$ \\
\hline
\end{tabular}

\section{Experiment No 2}

Consider the following nonlinear fractional pantograph equation with delay:

$$
\mathrm{D}^{\lambda} f(t)=1-2 f^{2}\left(\frac{1}{2} t\right), \quad 1<\lambda \leq 2, \quad \mathrm{f}(0)=1, \quad \mathrm{f}^{\prime}(0)=0
$$


Simulation of above nonlinear Pantograph equation by TMM with $\lambda=2$ and $\mathrm{k}=12$ gives the following series solution.

$$
f(t)=1-0.500004 t^{2}-0.00001851 t^{3}+0.042 t^{4}-0.00006 t^{5}-0.001 t^{6}+\ldots
$$

while the true solution of the above equation is given by $\cos (t)$. Figure 5 demonstrates the graphical comparison of true and TMM solution for $\lambda=2$ at $[0,6]$ Furthermore, solution at fractional values of $\lambda$ can also be visualized in Figure 5. Table 2 is displaying the comparison of the proposed scheme with some recent studies of Bernoulli and Laquerre wavelets methods while Table 3 is demonstrating the residual error that has been described through Eq. 10, for the above differential equation. Last row of Table 3 is reserved to represent the final values of MSE obtained after the learning process of unknown Taylor coefficients however corresponding to the MSE final values of unknown taylor coefficients can be envisioned in Figure 6. Data for 100 numbers of independent runs of algorithm for above delay differential equation can be visualized in Figure 7.

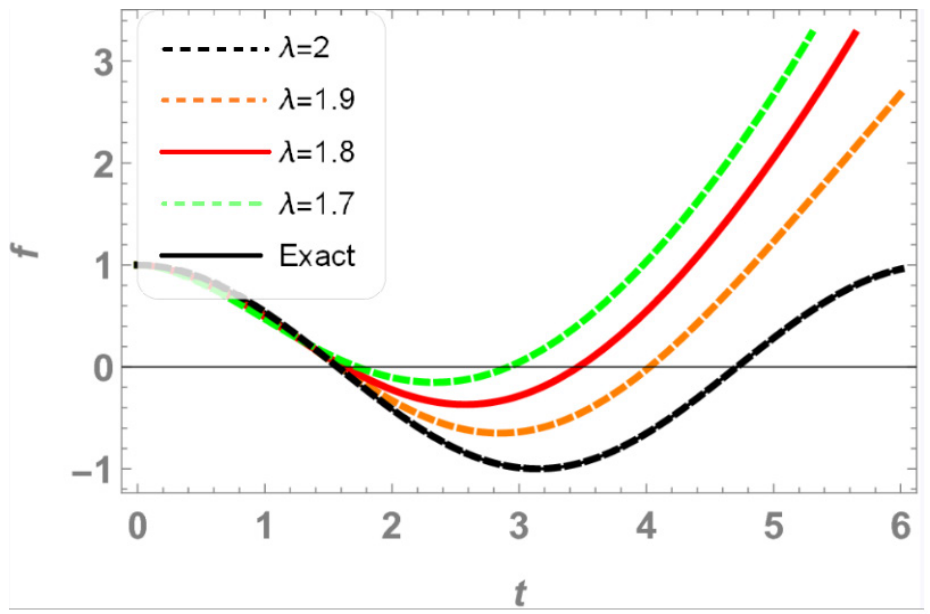

Figure 5. Solution of Example 2 at different values of $\lambda$. 

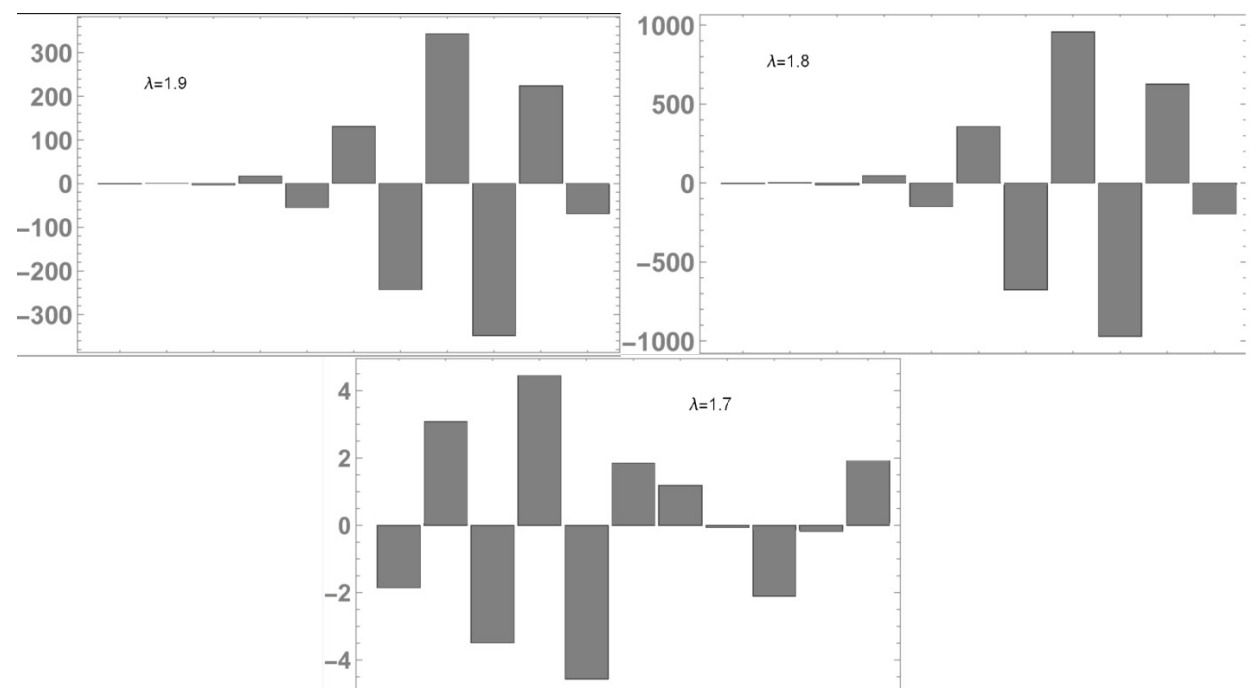

Figure 6. Final values of Unknown Taylor coefficients for example No 2 for $k=12$.
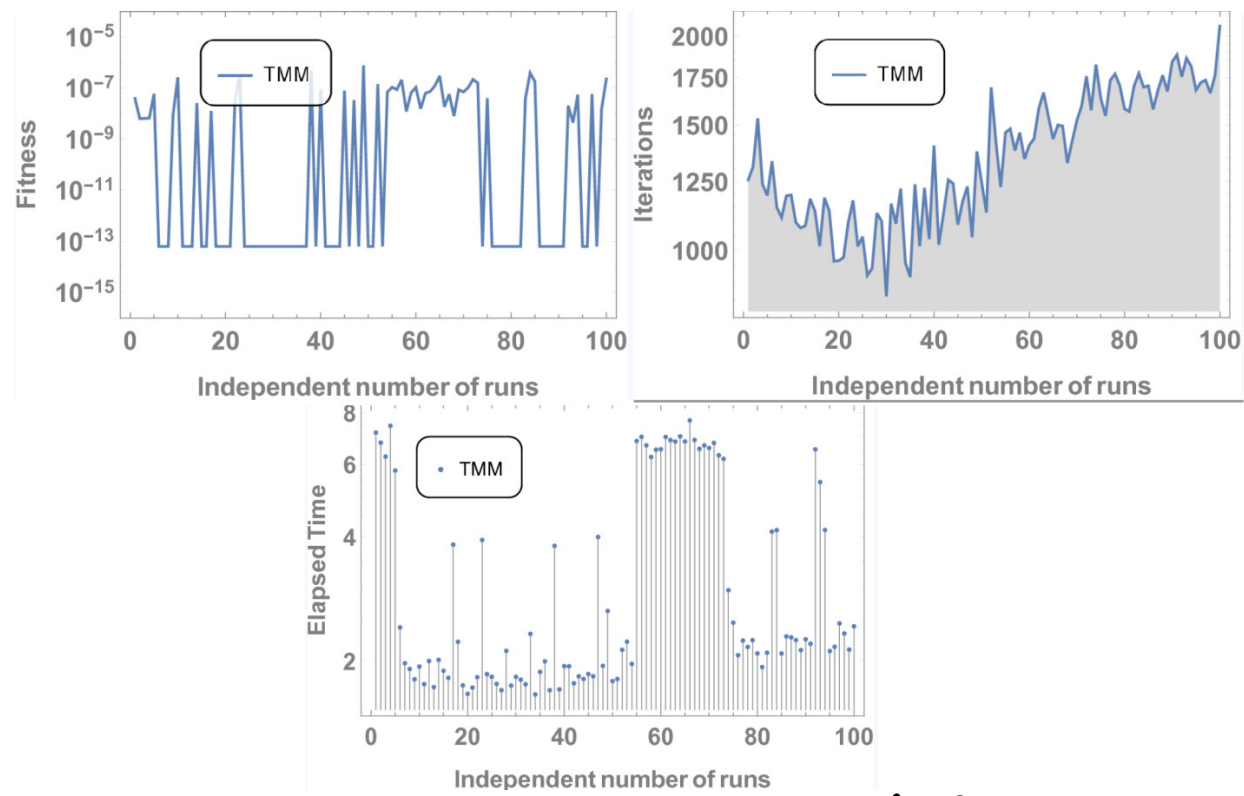

Figure 7. Data of 100 number of Independent runs of example No 2 for $\lambda=2$.

Table 2. Comparison of absolute error for example № 2 .

\begin{tabular}{|c|c|c|c|}
\hline$t$ & Absolute error & Absolute error & Absolute error \\
& $\lambda=2$ & $\lambda=1.9$ & $\lambda=1.8$ \\
\hline 0.1 & $2.2880 \times 10-8$ & - & $2.1000 \times 10-8$ \\
\hline 0.2 & $5.7153 \times 10-8$ & $5.78 \times 10-11$ & $2.0900 \times 10-8$ \\
\hline
\end{tabular}




\begin{tabular}{|c|c|c|c|}
\hline & Absolute error & Absolute error & Absolute error \\
\hline & $\lambda=2$ & $\lambda=1.9$ & $\lambda=1.8$ \\
\hline 0.3 & $8.4235 \times 10-8$ & - & $2.0900 \times 10-8$ \\
\hline 0.4 & $1.0627 \times 10-7$ & $6.25 \times 10-11$ & $2.0800 \times 10-8$ \\
\hline 0.5 & $1.3043 \times 10-7$ & - & $2.0600 \times 10-8$ \\
\hline 0.6 & $1.6246 \times 10-7$ & $3.01 \times 10-11$ & $2.0400 \times 10-8$ \\
\hline 0.7 & $2.0511 \times 10-7$ & - & $2.0300 \times 10-8$ \\
\hline 0.8 & $2.5853 \times 10-8$ & $2.62 \times 10-7$ & $2.0000 \times 10-8$ \\
\hline 1.0 & $3.2171 \times 10-7$ & - & $1.9900 \times 10-8$ \\
\hline
\end{tabular}

Table 3. Residual error for example No 2.

\begin{tabular}{|c|c|c|c|c|}
\hline & $\begin{array}{c}\text { Residual } \\
\text { error }\end{array}$ & $\begin{array}{c}\text { Residual } \\
\text { error }\end{array}$ & $\begin{array}{c}\text { Residual } \\
\text { error }\end{array}$ & $\begin{array}{c}\text { Residual } \\
\text { error }\end{array}$ \\
\hline & $\lambda=2$ & $\lambda=1.9$ & $\lambda=1.8$ & $\lambda=1.7$ \\
\hline 1 & $5.1393 \times 10-7$ & $1.4006 \times 10-3$ & $3.1913 \times 10-3$ & $1.8266 \times 10-2$ \\
\hline 2 & $1.3659 \times 10-7$ & $3.8499 \times 10-4$ & $1.2004 \times 10-3$ & $1.1830 \times 10-2$ \\
\hline 4 & $2.4985 \times 10-7$ & $2.1263 \times 10-4$ & $3.0410 \times 10-4$ & $8.0982 \times 10-3$ \\
\hline 5 & $3.1538 \times 10-7$ & $5.4257 \times 10-4$ & $1.2117 \times 10-3$ & $5.7085 \times 10-3$ \\
\hline 6 & $6.3298 \times 10-8$ & $5.3086 \times 10-4$ & $1.3318 \times 10-3$ & $6.7070 \times 10-3$ \\
\hline MSE & $4.0145 \times 10-8$ & $7.5912 \times 10-4$ & $1.7963 \times 10-3$ & $1.1744 \times 10-2$ \\
\hline
\end{tabular}

\section{Experiment No 3}

Consider the following nonlinear fractional pantograph differential equation with multiple proportional delays.

$$
\begin{gathered}
\mathrm{D}^{\lambda} f(t)=-f(t)-\left(\mathrm{D}^{\lambda} f\left(\frac{t}{3}\right)\right)^{3}+\mathrm{f}\left(\frac{\mathrm{t}}{2}\right)+2 \mathrm{t}+\frac{3}{4} \mathrm{t}^{2}+\frac{8}{7} \mathrm{t}^{3}, \quad 0<\lambda \leq 1, \\
f(0)=0
\end{gathered}
$$

By applying the TMM on above nonlinear pantograph fractional differential model, with fractional delay for $\lambda=1$ and $\mathrm{k}=3$, unknown coefficients are found to be $1.97151 \times 10^{-15}, 2$ and $4.79185 \times 10^{-16}$. By substituting these values in Eq. 2 the required solution is found to be $f(t)=1.97151 \times 10^{-15} t+t^{2}+7.98642 \times 10^{-17} t^{3}$ which is approximately equal to the true solution $f(t)=t^{2}$. For the fractional values of $\lambda$ the obtained MSE for the above example are found to be $2.64798 \times 10^{-7}, 2.3042 \times 10^{-6}$ and $1.24327 \times 10^{-6}$ for $\lambda=0.9,0.8$ and $0 . .7$ respectively. Figure 8 is demonstrating the solution at different values of $\lambda$ by proposed method with an exact solution at $\lambda=1$ while Figures 9 and 10 are 
representing the final values of Taylor coefficients and data of 100 number of independent runs respectively. Error analysis at different values of $\lambda$ on domain $t \in[0, h]$ can be observed in Figure 11 .

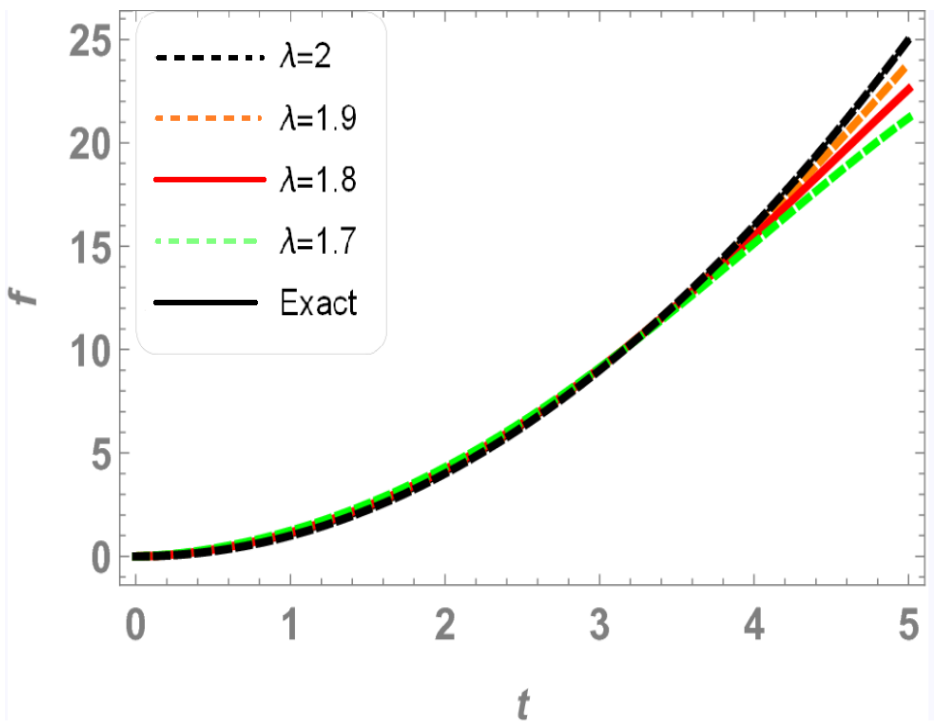

Figure 8. Solution of Example 3 at different values of $\lambda$.

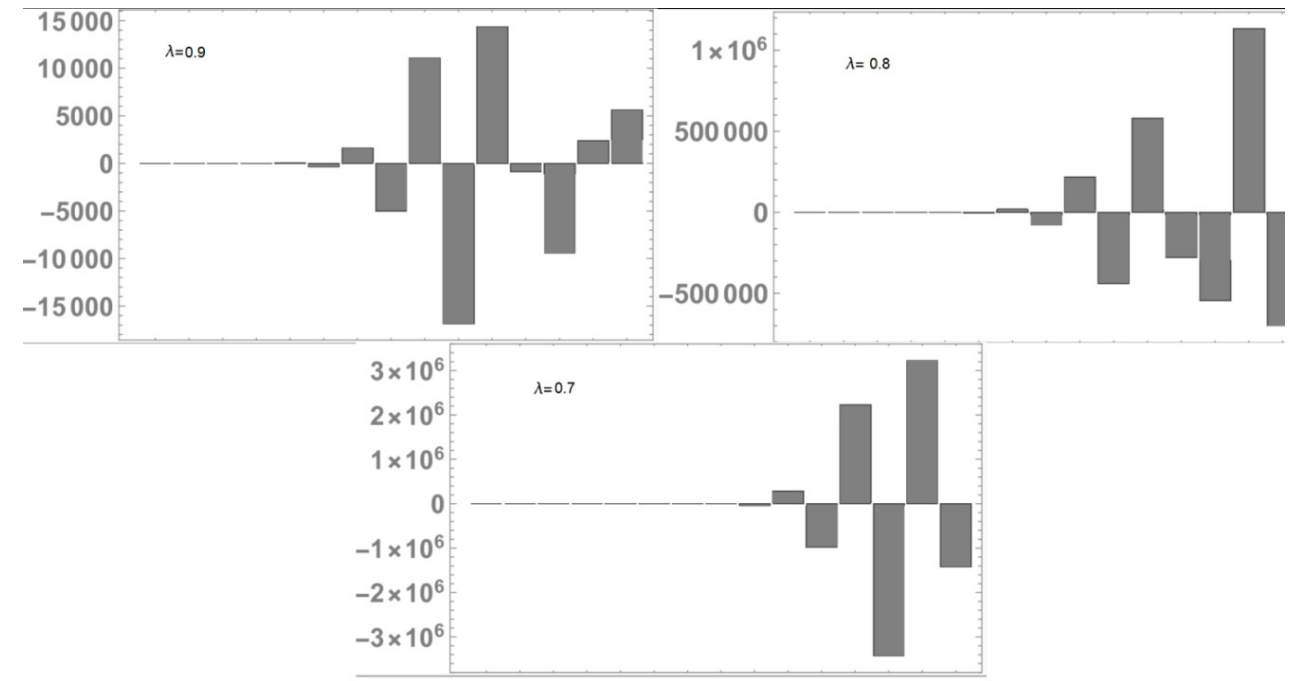

Figure 9. Final values of Unknown Taylor coefficients, for example, № 3 for $k=15$. 

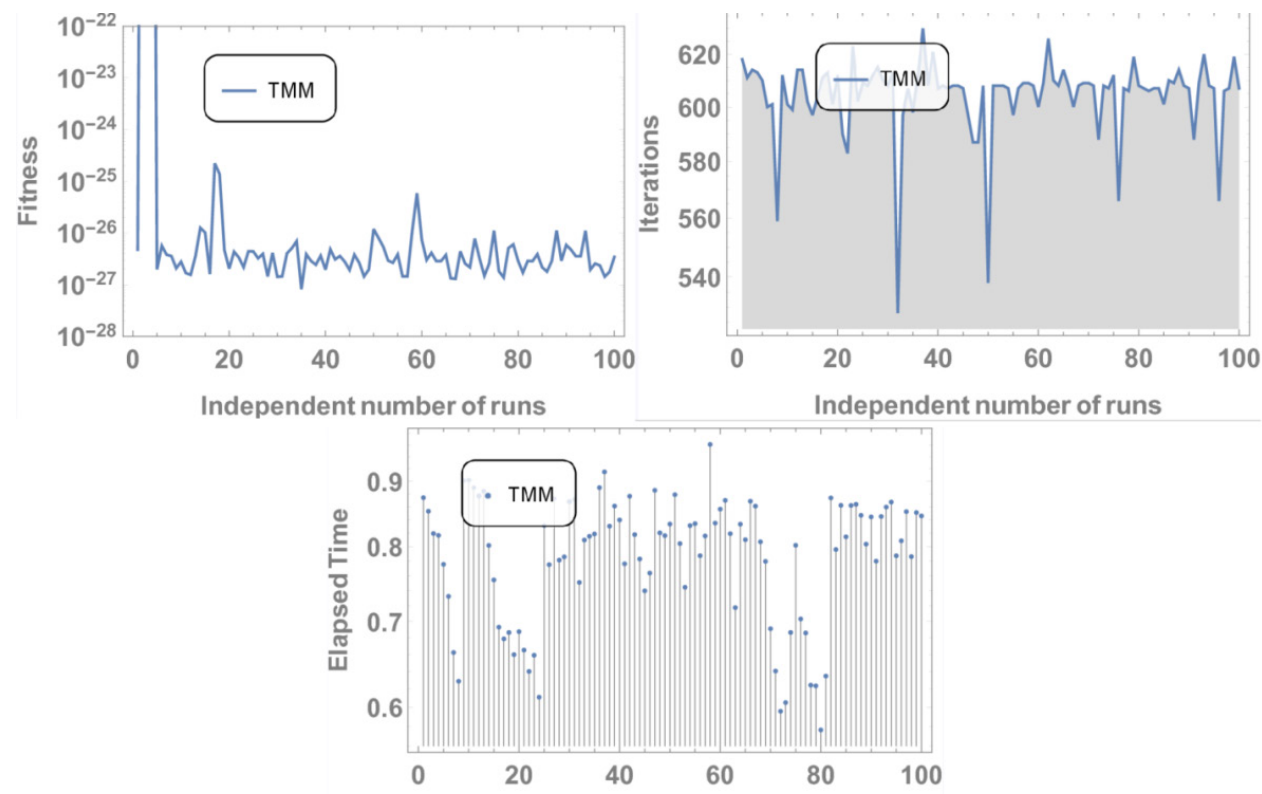

Independent number of runs

Figure 10. Data of 100 number of Independent runs of example No 3 for $\lambda=2$.

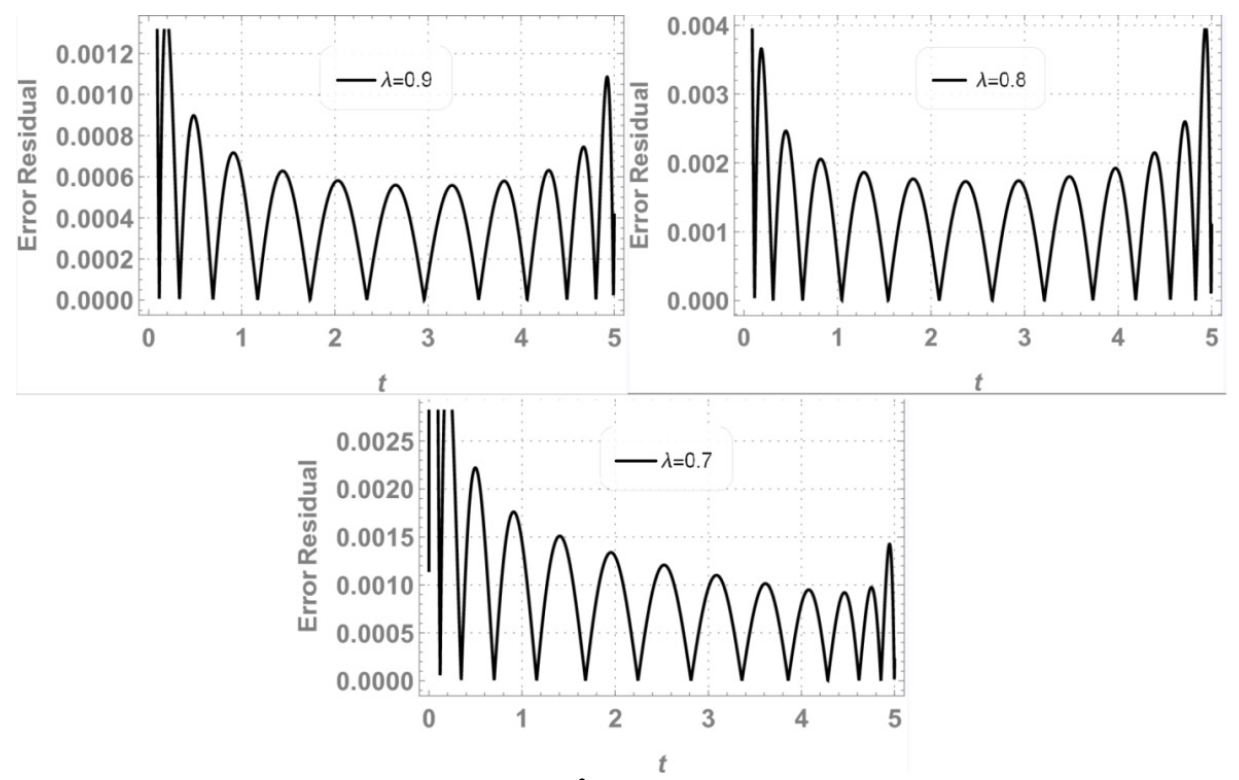

Figure 11. Residual error for fractional values $\lambda$, for example, № 3 . 


\section{Experiment No 4}

Consider the following nonlinear boundary value fractional pantograph differential equation with multiple proportional delays.

$$
\begin{gathered}
\mathrm{D}^{\lambda} f(t)=\left(f(t)^{2}+|f(t)|^{3}\right) f(t / 2), \quad 1<\lambda \leq 2, \\
f(0)=1 \text { and } f(1)=\frac{1}{2}
\end{gathered}
$$

By applying the TMM on above nonlinear boundary value pantograph fractional differential model, with fractional delay for $\lambda=2$ and $k=15$, results can be visualized in Figure 12. The true solution for $\lambda=1$ is given by $f(t)=\frac{1}{1+t}$. For the fractional values of $\lambda$ the obtained MSE for the above example are found to be $3.16567 \times 10^{-6}, 1.45514 \times 10^{-5}$ and $4.14856 \times 10^{-5}$ for $\lambda=0.9,0.8$ and $0 . .7$ respectively. Table 4 exhibits the comparison at different fractional values. Figure 12 is demonstrating the solution at different values of $\lambda$ by proposed method with an exact solution at $\lambda=2$ while Figures 13 and 14 are representing the final values of Taylor coefficients and data of 100 number of independent runs respectively.

Table 4. Absolute Error Comparison for experiment 4.

\begin{tabular}{|c|c|c|}
\hline$t$ & Absolute error & Absolute error \\
\hline 0.1 & $\lambda=2$ & $\lambda=1.9$ \\
\hline 0.2 & $8.0504 \times 10-6$ & $1.38 \times 10-4$ \\
\hline 0.3 & $5.0102 \times 10-6$ & $2.17 \times 10-4$ \\
\hline 0.4 & $5.3252 \times 10-6$ & $2.44 \times 10-4$ \\
\hline 0.5 & $3.5795 \times 10-6$ & $2.50 \times 10-4$ \\
\hline 0.6 & $1.1812 \times 10-6$ & $2.34 \times 10-4$ \\
\hline 0.7 & $7.5917 \times 10-7$ & $2.11 \times 10-4$ \\
\hline 0.8 & $1.5928 \times 10-6$ & $1.77 \times 10-5$ \\
\hline 0.9 & $1.2997 \times 10-6$ & $1.39 \times 10-5$ \\
\hline 1.0 & $1.0732 \times 10-7$ & $9.53 \times 10-5$ \\
\hline
\end{tabular}


Edición Especial Special Issue Mayo 2019

DOI: http://dx.doi.org/10.17993/3ctecno.2019.specialissue2.322-349

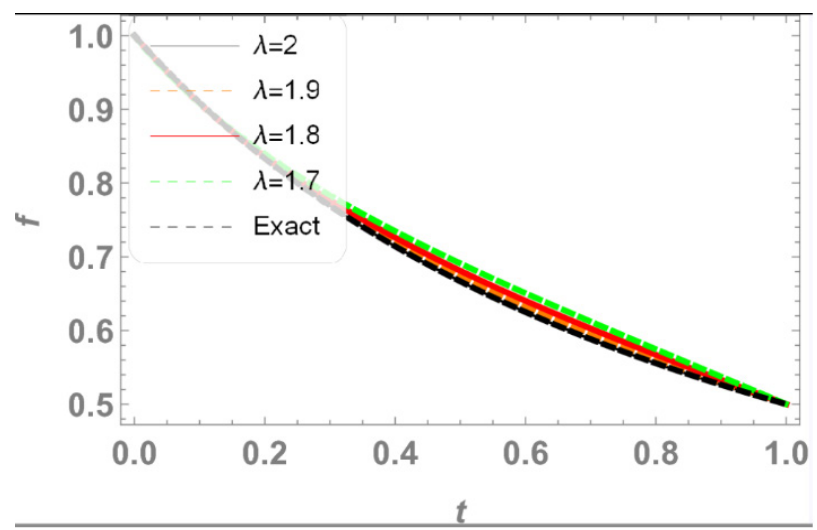

Figure 12. The solution of Example 4 at different values of $\lambda$.

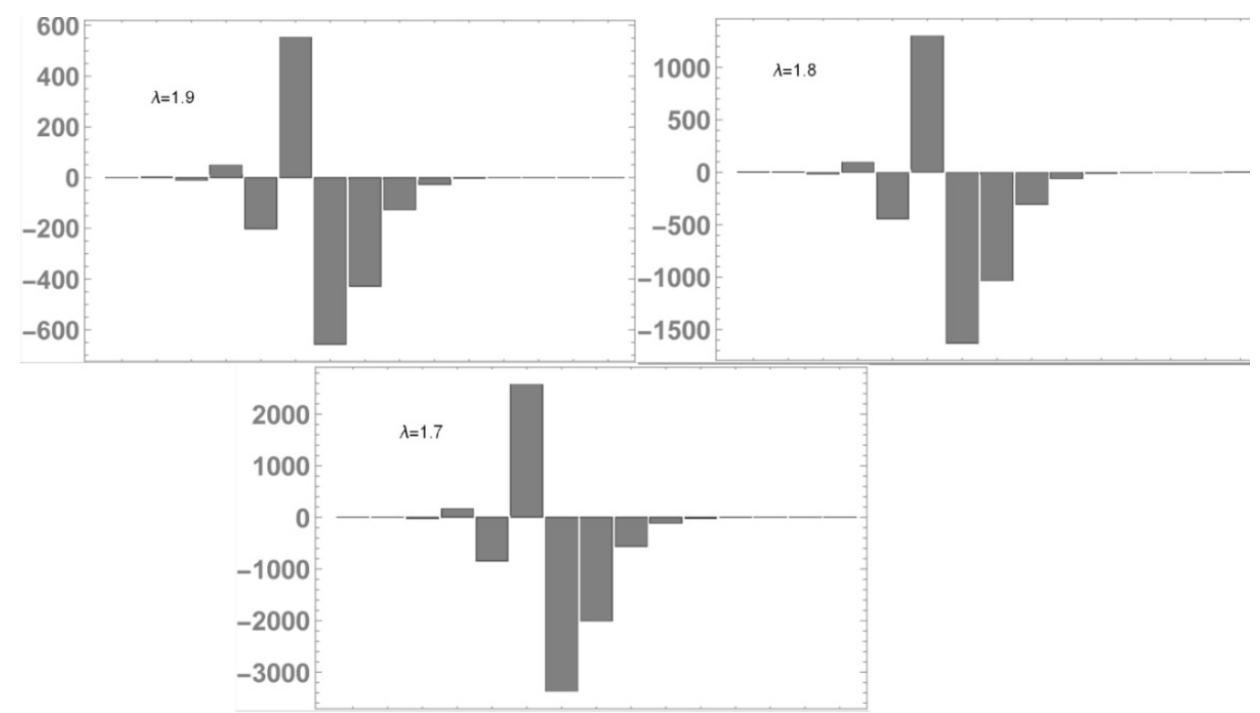

Figure 13. Final values of Unknown Taylor coefficients, for example, No 4 for $k=15$. 

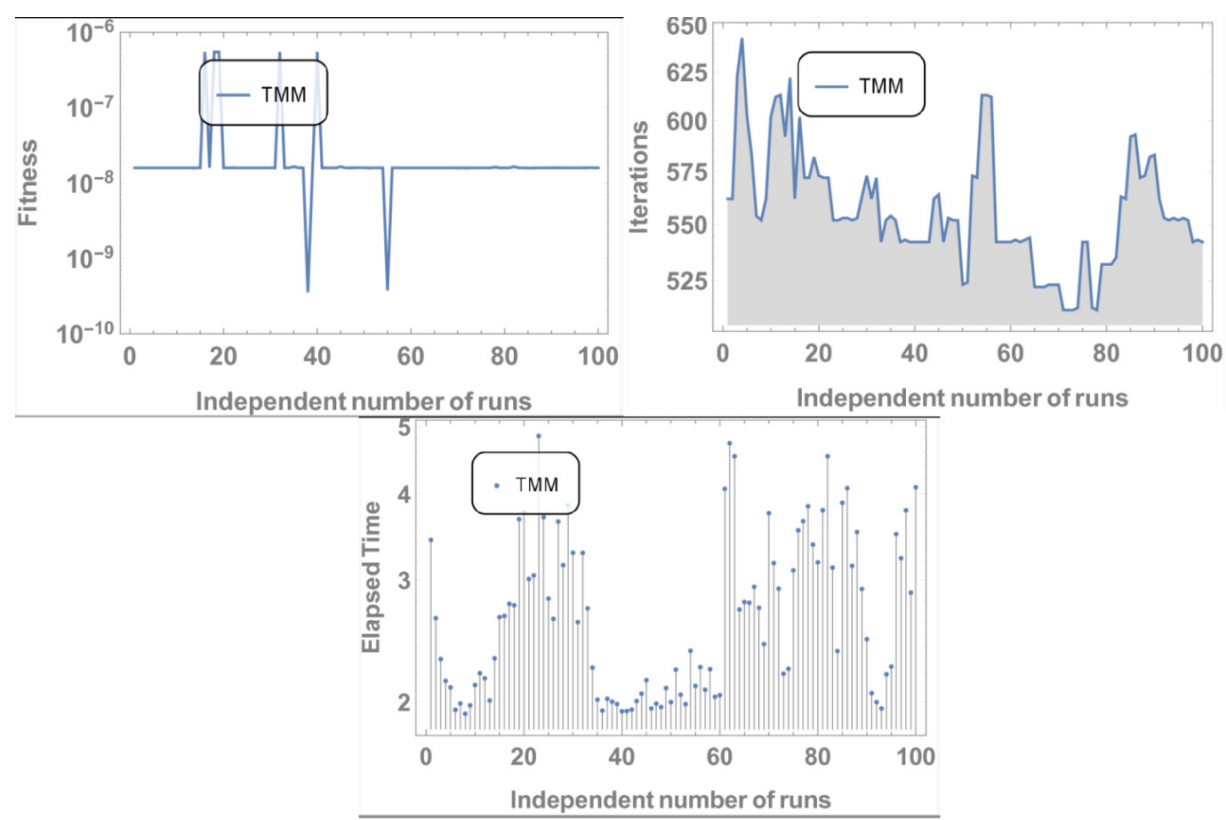

Figure 14. Data of 100 number of Independent runs of example No 4 for $\lambda=2$.

\section{DISCUSSION}

The effort, that is performed above is mainly dealt with the effective implementation of TMM on initial and boundary value fractional Pantograph differential equations with multiple proportional delays. To validate the strength of employed methodology some linear and nonlinear test experiments have been efficaciously simulated through TMM. Absolute and residual errors have been calculated to compare the effectiveness of TMM with other methods. Different type of data, related to Taylor coefficients and multiple runs of the procedure, has also been demonstrated above to make the procedure well comprehensible.

Considering the results of test experiment 1 , that is a linear initial value fractional Pantograph differential equation with single proportional delay, it is obvious that TMM has provided an excellent solution with $k=3$ and $\lambda=2$ that can be visualised through Figure 2 with overlapping lines of exact and TMM solution at $\lambda=2$. Values of residual error, which has been calculated according to the explained procedure in section 4 of this paper, is demonstrating prominent values 
ranged between $10^{-4}$ to $10^{-5}$ that, can be witnessed from Table 1 . Final values of unknown Taylor coefficients after learning through thermal minimization algorithm can be seen in Figure 3 while Figure 4 is establishing the results of multiple runs of an algorithm for fitness function, a number of iterations and elapsed time in seconds. It can be envisioned through Figure 4 that fitness function for experiment No 1 ranged between $10^{-26}-10^{-36}$, number of iterations during learning procedure by SA ranged between 100-400 iterations while elapsed time in seconds is less than 0.3 seconds with Core i 5 processor and 2 GB RAM.

Problem took for experiment No 2 is a nonlinear initial value fractional Pantograph differential equation with single proportional delay term. Solution by TMM simulation for experiment number 2 can be seen in Figure 5 at different values of $\lambda$ from which strength of proposed methodology can be observed. Comparison with a similar type of methods has been demonstrated in Table 2 that is witnessing method more powerful but here benefit of TMM is an effortless mechanism with the larger domain of implementation and less time consumption. Table 4 is depicting the values of residual error and MSE for fractional values of $\lambda$ that is demonstrating the ability of TMM to handle the nonlinear problems smoothly. Final values of unknown Taylor coefficients have been displayed in Figure 6 for the ease of reader moreover elapsed time for the above nonlinear problem is less than 8 seconds for all while for most of the time it is less than 3 seconds that can be witnessed through Figure 7. A number of iterations and fitness function are ranged $800-2000$ and $10^{-6}-10^{-13}$ respectively.

Experiment number 3 is showing a similar trend like the other two examples but this problem is a nonlinear fractional differential equation with multiple delays. TMM showed a promising solution for this experimental case that can be envisioned through Figure 8. Residual error for different fractional values of $\lambda$ has been presented in Figure 11 that is exhibiting the successful implementation of TMM. Time elapsed for this problem is under 0.9 seconds which is giving advantage to TMM over other methods to present effortless scheme with less time consumption. Other results are following the above trends. 
Experiment number 4 is a boundary value problem with nonlinear and delay terms. Solution provided by TMM can be seen in Figure 12 that is showing the efficacy of the proposed scheme. Comparison of the proposed scheme with a meta heuristic method can be seen in Table 4 but the author proposed the solution only for $\lambda=2$ while the current method has also simulated the problem for fractional values of $\lambda$. Elapsed time in seconds for this experiment is under five seconds with similar processor conditions as described for above problems that can be observed in Figure 14. Number of iterations during the learning process is varied between $500-650$.

\section{CONCLUSION}

In this effort, we studied the fractional pantograph equation via TMM. The accuracy and strength of the proposed method are ascertained by observing the error analysis for diverse numerical experiments. The obtained results show that linear and nonlinear neutral fractional Pantograph equations with proportional delays type differential equations can be cracked by this scheme with less effort and more accuracy. Results obtained from the above numerical experiments can be concluded as:

- For integer value of derivative TMM exhibits exceptionally excellent results that can be visualized in Figures 2, 5, 8 and 12.

- TMM converts the differential equations into a minimization problem so the accuracy of the method is merely dependent on obtained MSE or fitness function by implementation of SA and advancements in SA for better minimization can further develop the TMM for better results.

- Graphical representation of Error analysis for the above numerical experiments depicts that TMM can be employed on larger domains with accuracy.

- Challenging nonlinearities, boundary conditions and proportional delays can be handled with this scheme effortlessly.

- The proposed scheme can be further developed to obtain the solutions of complex differential models. 
- TMM can be employed to nonlinear FDEs without applying any perturbations and linearization.

\section{Data Availability Statement}

All data are provided in full in a different section of this paper. 


\section{REFERENCES}

Agarwal, R. P., Lakshmikantham, V. \& Nieto, J. J. (2010). On the concept of solution for fractional differential equations with uncertainty. Nonlinear Analysis: Theory, Methods $\mathcal{E}$ Applications, 72(6), pp. 2859-2862. doi: http://dx.doi. org/10.1016/j.na.2009.11.029

Arikoglu, A. \& Ozkol, I. (2007). Solution of difference equations by using differential transform method. Chaos, Solitons \& Fractals, 34(5), pp. 1473-1481. doi: http://dx.doi.org/10.1016/j.chaos.2006.09.004

Bai,Z. \& Lü,H. (2005). Positive solutions for boundary value problem of nonlinear fractional differential equation. Fournal of Mathematical Analysis and Applications, 311(2), pp. 495-505. doi: http://dx.doi.org/10.1016/j.jmaa.2005.02.052

Bhrawy, A. H., Al-Zahrani, A. A., Alhamed, Y. A. \& Baleanu, D. (2014). A new generalized Laguerre-Gauss collocation scheme for numerical solution of generalized fractional pantograph equations. Romanian Fournal of Physics, 59(7), pp. 646-657.

Chang, S. H. (2014). Taylor series method for solving a class of nonlinear singular boundary value problems arising in applied science. Applied Mathematics and Computation, 235, pp. 110-117. doi: http://dx.doi.org/10.1016/j. amc.2014.02.094

Diethelm, K., Ford, N. J. \& Freed, A. D. (2002). A predictor-corrector approach for the numerical solution of fractional differential equations. Nonlinear Dynamics, 29(1-4), pp. 3-22. doi: http://dx.doi.org/10.1023/A:101659221

Groza, G. \& Razzaghi, M. (2013). A Taylor series method for the solution of the linear initial-boundary-value problems for partial differential equations. Computers \& Mathematics with Applications, 66(7), pp. 1329-1343. doi: http://dx.doi. org/10.1016/j.camwa.2013.08.004 
Heydari, M., Loghmani, G. B. \& Hosseini, S. M. (2015). An improved piecewise variational iteration method for solving strongly nonlinear oscillators. Computational and Applied Mathematics, 34(1), pp. 215-249. doi: http://dx.doi. org/10.1007/s40314-014-0113-3

Iqbal, M. A., Saeed, U. \& Mohyud-Din, S. T. (2015). Modified Laguerre wavelets method for delay differential equations of fractional-order. Egyptian Fournal of Basic Applied Science, 2, pp. 50-54. doi: http://dx.doi.org/10.1016/j. ejbas.2014.10.004

Isah, A., Phang, G. \& Phang, P. (2017). Collocation Method Based on Genocchi Operational Matrix for Solving Generalized Fractional Pantograph Equations. International Fournal of Differential Equations, (7-8), pp. 1-10. doi: http:// dx.doi.org/10.1155/2017/2097317

Kaplan, T., Gray, L. J. \& Liu, S. H. (1987). Self-affine fractal model for a metal-electrolyte interface. Physical Review B, 35(10), p. 5379. doi: http://dx.doi. org/10.1103/PhysRevB.35.5379

Kirkpatrick, S., Gelatt, G. D. \& Vecchi, M. P. (1983). Optimization by simulated annealing. Science, 220(4598), pp. 671-680. doi: http://dx.doi. org/10.1126/science.220.4598.671

Kulish, V. V. \& Lage, J. L. (2002). Application of fractional calculus to fluid mechanics. Fournal of Fluids Engineering, 124(3), pp. 803-806. doi: http://dx.doi. org/10.1115/1.1478062

Le Mehaute, A. \& Crepy, G. (1983). Introduction to transfer and motion in fractal media: the geometry of kinetics. Solid State Ionics, 9, pp. 17-30. Dio: 10.1016/0167-2738(83)90207-2

Magin, R. L. \& Ovadia, M. (2008). Modeling the cardiac tissue electrode interface using fractional calculus. Fournal of Vibration and Control, 14(9-10), pp. 1431-1442. doi: http://dx.doi.org/10.1177/1077546307087439

Malik, S. G. \& Arora, S. (1992). Mathematical analysis. New Age International. 
Marzban, H. R. \& Razzaghi, M. (2006). Solution of multi-delay systems using hybrid of block-pulse functions and Taylor series. Fournal of Sound and Vibration, 292(3), pp. 954-963. doi: http://dx.doi.org/10.1016/j.jsv.2005.08.007

Rahimkhani, P., Ordokhani, Y. \& Babolian, E. (2017). Numerical solution of fractional pantograph differential equations by using generalized fractionalorder Bernoulli wavelet. Fournal of Computational and Applied Mathematics, 309, pp. 493-510. doi: http://dx.doi.org/10.1016/j.cam.2016.06.005

Rahimkhani, P., Ordokhani, Y. \& Babolian, E. (2018). Müntz-Legendre wavelet operational matrix of fractional-order integration and its applications for solving the fractional pantograph differential equations. Numerical Algorithms, 77(4), pp. 1283-1305. doi: http://dx.doi.org/10.1007/s1 1075-017-0363-4

Raja, M. A. Z. (2014). Numerical treatment for boundary value problems of pantograph functional differential equation using computational intelligence algorithms. Applied Soft Computing, 24, pp. 806-821. doi: http://dx.doi. org/10.1016/j.asoc.2014.08.055

Razzaghi, M. \& Razzaghi, M. (1989). Taylor series analysis of time-varying multi-delay systems. International Fournal of Control, 50(1), pp. 183-192. doi: http:// dx.doi.org/10.1080/00207178908953357

Robinson, D. A. (1981). The use of control systems analysis in the neurophysiology of eye movements. Annual review of neuroscience, 4(1), pp. 463-503. doi: http:// dx.doi.org/10.1146/annurev.ne.04.030181.002335

Saadatmandi, A. \& Dehghan, M. (2009). Variational iteration method for solving a generalized pantograph equation. Computers $\&$ Mathematics with Applications, 58(11-12), pp. 2190-2196. doi: http://dx.doi.org/10.1016/j. camwa.2009.03.017 
Sebaa, N., Fellah, Z. E. A., Fellah, M., Ogam, E., Wirgin, A., Mitri, F. G., ... Lauriks, W. (2006). Ultrasonic characterization of human cancellous bone using the Biot theory: Inverse problem. The Fournal of the Acoustical Society of America, 120(4), pp. 1816-1824. doi: http://dx.doi.org/10.1121/1.2335420

Syam, M. I. \& Jaradat, H. M. (2017). An Accurate Integral Solution for Solving the Pantograph Equation. International fournal of Applied and Computational Mathematics, 3(1), pp. 925-935. doi: http://dx.doi.org/10.1007/s40819-0170390-9 